doi:10.13108/2020-12-3-44

\title{
ON FAMILY OF COMPLEX STRAIGHT LINES SUFFICIENT FOR EXISTENCE OF HOLOMORPHIC CONTINUATION OF CONTINUOUS FUNCTIONS ON BOUNDARY OF DOMAIN
}

\author{
A.M. KYTMANOV, S.G. MYSLIVETS
}

\begin{abstract}
The problem on holomorphic continuation of functions defined on the boundary of a domain into this domain is topical in the multi-dimensional complex analysis. It has a long history beginning from works by Poincaré and Hartogs. In the present work we consider continuous functions defined on a boundary of a bounded domain $D$ in $\mathbb{C}^{n}$, $n>1$, and possessing a generalized Morera property along the family of complex straight lines intersecting the germ of a real analytic manifold of codimension 2 lying away of the boundary of the domain. The Morera property is the vanishing of the integral of this function over the intersection of the boundary of the domain with the complex curve. We show that such function possesses a holomorphic continuation into the domain $D$. For functions of one complex variable, the Morera property obviously does not imply the existence of holomorphic continuation. This is why such problem can be considered only in the multi-dimensional case $(n>1)$.
\end{abstract}

Keywords: holomorphic continuation, Morera boundary condition, Bochner-Martinelli kernel.

Mathematics Subject Classification: 34A10, 32A26, 32D15

\section{INTRODUCTION}

The paper provides some results on holomorphic continuation of functions continuous on the boundary of a bounded domain into this domain. We discuss the functions satisfying Morera boundary condition. Its matter is the vanishing of the integrals of this functions over the intersection of the boundary of the domain with complex straight lines or complex planes. E. Grinberg [1] studied the functions with Morera property in a ball, in fact, this result contained already in paper by M.L. Agranovsky and R.E.Valskii [2]. I. Globevnik and E.E. Stout [3] obtained a boundary Morera theorem for an arbitrary bounded domain with a twice smooth boundary. A local variant of Morera theorem was considered by J. Globevnik [4], D. GovekarLebo [5]. In work [6], S.G. Myslivets considered functions with Morera property along complex curves, while in works [7], 8], [9] were provided some families of complex curves sufficient for holomorphic continuation of the functions.

As a sufficient set we consider the set of complex straight lines intersecting the germ of a real-analytic manifold of real dimension $(2 n-2)$ disjoint with the boundary of the domain $D$.

Let $D \subset \mathbb{C}^{n},(n>1)$, be a bounded domain with a connected boundary of class $\mathcal{C}^{2}$ of form

$$
D=\left\{z \in \mathbb{C}^{n}: \rho(z)<0\right\},
$$

A.M. Kytmanov, S.G. Myslivets, On family of COMPlex Straight Lines Sufficient for exisTENCE OF HOLOMORPHIC CONTINUATION OF CONTINUOUS FUNCTIONS ON BOUNDARY OF DOMAIN.

(C) Kytmanov A.M., Myslivets S.G. 2020.

The research supported by Russian Foundation for Basic Researches under project no. 18-51-41011-Uzbt.

Submitted February 8, 2020. 
where $\rho(z)$ is a smooth function of class $\mathcal{C}^{2}$ being real in the vicinity of the set $\bar{D}$ such that $\left.d \rho\right|_{\partial D} \neq 0$. We identify $\mathbb{C}^{n}$ with $\mathbb{R}^{2 n}$ as follows: $z=\left(z_{1}, \ldots, z_{n}\right)$, where $z_{j}=x_{j}+i y_{j}, x_{j}, y_{j} \in \mathbb{R}$, $j=1, \ldots, n$.

We consider complex straight lines $l_{z, b}$ of the form

$$
l_{z, b}=\left\{\zeta \in \mathbb{C}^{n}: \zeta_{j}=z_{j}+b_{j} t, j=1, \ldots, n, t \in \mathbb{C}\right\}
$$

passing through the point $z \in \mathbb{C}^{n}$ along the vector $b=\left\{b_{1}, \ldots, b_{n}\right\} \in \mathbb{C P}^{n-1}$; the direction $b$ is defined up to a multiplication of a complex number $\lambda \neq 0$.

Definition 1. A continuous function $f$ on $\partial D(f \in \mathcal{C}(\partial D))$ satisfies Morera property along a complex plane $l$ of dimension $k, 1 \leqslant k \leqslant n-1$, if

$$
\int_{\partial D \cap l} f(\zeta) \beta(\zeta)=0
$$

for each differential form $\beta$ of type $(k, k-1)$ with constant coefficients.

The plane $l$ is assumed to intersect transversally the boundary of the domain $D$.

If $l_{z, b}$ is a complex straight line intersecting $\partial D$ transversally, then the Morera property along $l_{z, b}$ is

$$
\int_{\partial D \cap l_{z, b}} f(z+b t) d t=\int_{\partial D \cap l_{z, b}} f\left(z_{1}+b_{1} t, \ldots, z_{n}+b_{n} t\right) d t=0
$$

for a given parametrization $\zeta=z+b t$ along a complex straight line $l_{z, b}$.

For complex straight lines we consider a more general condition.

Let $m$ be a fixed nonnegative integer, then the condition

$$
\int_{\partial D \cap l_{z, b}} f(z+b t) t^{m} d t=\int_{\partial D \cap l_{z, b}} f\left(z_{1}+b_{1} t, \ldots, z_{n}+b_{n} t\right) t^{m} d t=0
$$

is called a generalized Morera condition along the complex straight line $l_{z, b}$. As $m=0$, condition (3) becomes condition (2).

Let $\Gamma$ be the germ of a real-analytic manifold of real dimension $(2 n-2)$. We assume that $0 \in \Gamma$ and in some neighbourhood of the origin the manifold $\Gamma$ is of the form

$$
\Gamma=\left\{\zeta \in \mathbb{C}^{n}: \Phi(\zeta)+i \Psi(\zeta)=0\right\}
$$

where $\Phi, \Psi$ are real-analytic functions real-valued in the vicinity of the point. Here $\zeta=$ $\left(\zeta_{1}, \ldots, \zeta_{n}\right)$ and $\zeta_{j}=\xi_{j}+i \eta_{j}, \xi_{j}, \eta_{j} \in \mathbb{R}, j=1, \ldots, n$. The smoothness condition of the manifold $\Gamma$ is

at each point $\zeta \in \Gamma$.

$$
\operatorname{rank} A=\operatorname{rank}\left(\begin{array}{cccccc}
\frac{\partial \Phi}{\partial \xi_{1}} & \ldots & \frac{\partial \Phi}{\partial \xi_{n}} & \frac{\partial \Phi}{\partial \eta_{1}} & \ldots & \frac{\partial \Phi}{\partial \eta_{n}} \\
\frac{\partial \Psi}{\partial \xi_{1}} & \cdots & \frac{\partial \Psi}{\partial \xi_{n}} & \frac{\partial \Psi}{\partial \eta_{1}} & \cdots & \frac{\partial \Psi}{\partial \eta_{n}}
\end{array}\right)=2
$$

We consider complex straight lines of form (1) and we let $b_{j}=c_{j}+i d_{j}, c_{j}, d_{j} \in \mathbb{R}, j=1, \ldots, n$ and $t=u+i v, u, v \in \mathbb{R}$. Then in terms of real coordinates the straight lines $l_{z, b}$ are defined as follows:

$$
l_{z, b}=\left\{\xi, \eta \in \mathbb{R}^{n}: \xi_{j}=x_{j}+c_{j} u-d_{j} v, \eta_{j}=y_{j}+d_{j} u+c_{j} v, j=1, \ldots, n\right\} .
$$

We recall Lemma 1 from [8]. 
Lemma 1. Let a vector $b^{0}=\left(b_{1}^{0}, \ldots, b_{n}^{0}\right) \in \mathbb{C P}^{n-1}$ be such that $D \cap l_{0, b^{0}} \neq \varnothing$. Then there exists $\varepsilon>0$ such that for all $z$ obeying $|z|<\varepsilon$ and all $b$ such that $\left|b-b^{0}\right|<\varepsilon$, the following intersections are non-empty: $D \cap l_{z, b} \neq \varnothing$ and $\Gamma \cap l_{z, b} \neq \varnothing$.

We shall also need Lemma 2 from [8].

Lemma 2. Let for some $z$ and all $\zeta, b$ such that $D \cap l_{z, b} \neq \varnothing$, for $\zeta \in \partial D \cap l_{z, b}$, the function $\rho$ defining the domain $D$ satisfies the conditions

$$
\sum_{j=1}^{n} \frac{\partial \rho}{\partial \zeta_{j}} b_{j} \neq 0
$$

Then the curves $\partial D \cap l_{z, b}$ are smooth and depend analytically on the parameter $b$.

For instance, the assumptions of Lemma 2 are satisfied by domains in $\mathbb{C}^{n}$ strongly star-shaped with respect to a point $z \in D$, strongly convex, and strongly linear convex.

Theorem 1. Let a bounded domain $D \subset \mathbb{C}^{n}$ with a connected smooth boundary of class $\mathcal{C}^{2}$ satisfy conditions (5) for the points z lying in the vicinity of the manifold $\Gamma$ such that $\partial D \cap \Gamma=\varnothing$. Let a function $f \in \mathcal{C}(\partial D)$ satisfy generalized Morera conditions (3), that is,

$$
\int_{\partial D \cap l_{z, b}} f\left(z_{1}+b_{1} t, \ldots, z_{n}+b_{n} t\right) t^{m} d t=0
$$

for each $z \in \Gamma, b \in \mathbb{C P}^{n-1}$ and a fixed integer nonnegative number $m$. Then the function $f$ possesses a holomorphic continuation into the domain $D$.

Proof. We consider Bochner-Martinelli kernel

$$
U(\zeta, z)=\frac{(n-1) !}{(2 \pi i)^{n}} \sum_{k=1}^{n}(-1)^{k-1} \frac{\bar{\zeta}_{k}-\bar{z}_{k}}{|\zeta-z|^{2 n}} d \bar{\zeta}[k] \wedge d \zeta
$$

As it is known, in coordinates $b$ and $t$, the kernel $U(\zeta, z)$ reads as [9, Lm. 39.1]:

$$
U(\zeta, z)=\lambda(b) \wedge \frac{d t}{t}
$$

where $\lambda(b)$ is a differential form of type $(n-1, n-1)$ in $\mathbb{C P}^{n-1}$ independent of $t$, while $z \notin \partial D$.

We consider an integral

$$
M_{\alpha} f(z)=\int_{\partial D_{\zeta}}(\zeta-z)^{\alpha} f(\zeta) U(\zeta, z)
$$

where $\alpha=\left(\alpha_{1}, \ldots, \alpha_{n}\right)$ is an arbitrary multi-index such that

$$
\|\alpha\|=\alpha_{1}+\ldots+\alpha_{n}=m+1
$$

and

$$
(\zeta-z)^{\alpha}=\left(\zeta_{1}-z_{1}\right)^{\alpha_{1}} \cdots\left(\zeta_{n}-z_{n}\right)^{\alpha_{n}}
$$

By the Fubini theorem and the form of the kernel we obtain:

$$
M_{\alpha} f(z)=\int_{\mathbb{C P}^{n-1}} b^{\alpha} \lambda(b) \int_{\partial D \cap l_{z, b}} f\left(z_{1}+b_{1} t, \ldots, z_{n}+b_{n} t\right) t^{m} d t .
$$


By the assumptions and Lemma 1, the identities

$$
\int_{\partial D \cap l_{z, b}} f\left(z_{1}+b_{1} t, \ldots, z_{n}+b_{n} t\right) t^{m} d t=0
$$

hold for all sufficiently small $z$ and for $b$ in $\mathbb{C P}^{n-1}$. Then

$$
M_{\alpha} f(z)=\int_{\partial D_{\zeta}}(\zeta-z)^{\alpha} f(\zeta) U(\zeta, z) \equiv 0
$$

for all $z$ such that $|z|<\varepsilon$.

We rewrite a function $M_{\alpha} f(z)$ in another form. We consider differential forms $U_{s}(\zeta, z)$ :

$$
\begin{aligned}
U_{s}(\zeta, z)=\frac{(-1)^{s}(n-2) !}{(2 \pi i)^{n}}( & \sum_{j=1}^{s-1}(-1)^{j} \frac{\bar{\zeta}_{j}-\bar{z}_{j}}{|\zeta-z|^{2 n-2}} d \bar{\zeta}[j, s] \\
& \left.+\sum_{j=s+1}^{n}(-1)^{j-1} \frac{\bar{\zeta}_{j}-\bar{z}_{j}}{|\zeta-z|^{2 n-2}} d \bar{\zeta}[s, j]\right) \wedge d \zeta .
\end{aligned}
$$

It is easy to confirm that

$$
\bar{\partial}\left(\frac{1}{\zeta_{s}-z_{s}} U_{s}(\zeta, z)\right)=U(\zeta, z)
$$

as $\zeta_{s} \neq z_{s}, s=1, \ldots, n$. Then condition (6) can be rewritten as

$$
\int_{\partial D_{\zeta}} f(\zeta) \bar{\partial}\left((\zeta-z)^{\beta} U_{s}(\zeta, z)\right) \equiv 0
$$

for $z$ such that $|z|<\varepsilon$ and for all monomials $(\zeta-z)^{\beta}$ with $\|\beta\|=m$.

We are going to show that condition (7) holds also for monomials $(\zeta-z)^{\gamma}$ with $\|\gamma\|<m$. Indeed, we consider a monomial $(\zeta-z)^{\gamma}$ with $\|\gamma\|=m-1$. Then condition (7) holds for the monomials of form:

$$
(\zeta-z)^{\beta}\left(\zeta_{k}-z_{k}\right), \quad k=1, \ldots, n,
$$

since the degree of these monomials is equal to $m$.

The identity holds:

$$
\begin{aligned}
\frac{\partial}{\partial \zeta_{k}}\left((\zeta-z)^{\gamma}\left(\zeta_{k}-z_{k}\right) U_{s}(\zeta, z)\right)= & \left(\gamma_{k}+1\right)(\zeta-z)^{\gamma} U_{s}(\zeta, z) \\
& -(n-1)(\zeta-z)^{\gamma} \frac{\left(\zeta_{k}-z_{k}\right)\left(\bar{\zeta}_{k}-\bar{z}_{k}\right)}{|\zeta-z|^{2}} U_{s}(\zeta, z) .
\end{aligned}
$$

Summing up identities (8) over $k$, we obtain:

$$
\sum_{k=1}^{n} \frac{\partial}{\partial \zeta_{k}}\left((\zeta-z)^{\gamma}\left(\zeta_{k}-z_{k}\right) U_{s}(\zeta, z)\right)=(\|\gamma\|+1)(\zeta-z)^{\gamma} U_{s}(\zeta, z) .
$$

Since condition (7) can be differentiated in $z$ as $|z|<\varepsilon$, and the derivatives of expression (9) in $z$ and $\zeta$ differ only by the sign, it follows from (9) that the degree of the monomial in (7) can be lessen by one. Lessening successively this degree by one, we arrive at the conditions:

$$
\int_{\partial D_{\zeta}} f(\zeta) \bar{\partial} U_{s}(\zeta, z) \equiv 0
$$


for $|z|<\varepsilon$ and $s=1, \ldots, n$, that is,

$$
\int_{\partial D_{\zeta}}\left(\zeta_{s}-z_{s}\right) f(\zeta) U(\zeta, z) \equiv 0
$$

for $|z|<\varepsilon$ and $s=1, \ldots, n$.

Applying the Laplace operator to the left hand side of identity $(10)$

$$
\Delta=\frac{\partial^{2}}{\partial z_{1} \partial \bar{z}_{1}}+\ldots+\frac{\partial^{2}}{\partial z_{n} \partial \bar{z}_{n}},
$$

we obtain that

$$
\frac{\partial}{\partial \bar{z}_{s}} \int_{\partial D_{\zeta}} f(\zeta) U(\zeta, z) \equiv 0
$$

for $|z|<\varepsilon$ and $s=1, \ldots, n$. Here we have employed the harmonicity of the kernel $U(\zeta, z)$ and the identity

$$
\Delta(g h)=h \Delta g+g \Delta h+\sum_{j=1}^{n} \frac{\partial g}{\partial \bar{z}_{j}} \frac{\partial h}{\partial z_{j}}+\sum_{j=1}^{n} \frac{\partial g}{\partial z_{j}} \frac{\partial h}{\partial \bar{z}_{j}} .
$$

Hence, Bochner-Martinelli integral of the function $f$, namely,

$$
M f(z)=\int_{\partial D_{\zeta}} f(\zeta) U(\zeta, z)
$$

is a function holomorphic in the vicinity of the origin.

If $\Gamma \subset \mathbb{C}^{n} \backslash \bar{D}$, then $M f(z) \equiv 0$ outside $\bar{D}$ since the boundary is connected and $M f(z)$ tends to zero as $|z| \rightarrow \infty$ and then the function $f$ is continued holomorphically in the domain $D$ [10, Cor. 15.5].

If $\Gamma \subset D$, then the function $M f$ is holomorphic in $D$ and the boundary values of $M f$ coincides with $f[10$, Cor. 15.6].

The proof of Theorem 1 follows the main lines of Theorem 3 in [8] but there is a series of differences related with the continuity of the function.

As $m=0$, conditions (3) become the Morera boundary condition [3]:

$$
\int_{\partial D \cap l_{z, b}} f\left(z_{1}+b_{1} t, \ldots, z_{n}+b_{n} t\right) d t=0 .
$$

Corollary 1. Let a domain D satisfies the assumptions of Theorem 1, while the function $f \in \mathcal{C}(\partial D)$ satisfies condition (11) for all $z \in \Gamma$ and $b \in \mathbb{C P}^{n-1}$. Then the function $f$ can be holomorphically continued into $D$.

\section{BIBLIOGRAPHY}

1. Grinberg E.A. A boundary analogue of Morera's theorem in the unit ball of $\mathbb{C}^{n} / /$ Proc. Amer. Math. Soc. 102:1, 114-116 (1988).

2. M.L. Agranovskii, R.E. Valskii. Maximality of invariant algebras of functions // Sibir. Matem. Zhurn. 12:1, 3-12 (1971). [Siberian Math. J. 12:1, 1-7 (1971).]

3. J. Globevnik, E.L. Stout. Boundary Morera theorems for holomorphic functions of several complex variables // Duke Math. J. 64:3, 571-615 (1991).

4. J. Globevnik. A boundary Morera theorem // J. Geom. Anal. 3:3, 269-277 (1993).

5. D. Govekar-Leban. Local boundary Morera theorems // Math. Z. 233, 265-286 (2000). 
6. S.G. Myslivets. On a boundary version of Morera's theorem // Sibir. Matem. Zhurn. 42:5, 11361146 (2001). [Siberian Math. J.42:5, 952-960 (2001).]

7. A.M. Kytmanov, S.G. Myslivets. On families of complex lines sufficient for holomorphic extension // Matem. Zamet. 83:4, 545-551 (2008). [Math. Notes. 83:4, 500-505 (2008).]

8. A.M. Kytmanov, S.G. Myslivets. Some families of complex lines sufficient for holomorphic continuation of functions // Izv. VUZov. Matem. 4, 72-80 (2011). [Russian Math. (Iz. VUZ). 55:4, 60-66 (2011).]

9. A.M. Kytmanov, S.G. Myslivets. Multidimensional integral representations. Problems of analytic continuation. Springer Verlag, Basel (2015).

10. A.M. Kytmanov. The Bochner-Martinelli integral and its applications. Nauka, Novosibirsk (1992). [Birkhäuser, Basel (1995).]

Alexander Mechislavovich Kytmanov, Siberian Federal University,

Svobodny av. 79, 660041, Krasnoyarsk

E-mail: akytmanov@sfu-kras.ru

Simona Glebovna Myslivets,

Siberian Federal University,

Svobodny av. 79, 660041, Krasnoyarsk

E-mail: smyslivets@sfu-kras.ru 